\title{
BMJ Open Impact of the Early Start Denver Model on the cognitive level of children with autism spectrum disorder: study protocol for a randomised controlled trial using a two-stage Zelen design
}

Sandrine Touzet, ${ }^{1,2}$ Pauline Occelli, ${ }^{1,2}$ Carmen Schröder, ${ }^{3,4}$ Sabine Manificat, ${ }^{5}$ Ludovic Gicquel, ${ }^{6,7}$ Razvana Stanciu, ${ }^{8}$ Marie Schaer, ${ }^{9}$ Marie-Joelle Oreve, ${ }^{10}$ Mario Speranza, ${ }^{10,11}$ Angelique Denis, ${ }^{1,2}$ Amelie Zelmar, ${ }^{1,2}$ Bruno Falissard, ${ }^{12,13}$ Nicolas Georgieff, ${ }^{14,15}$ Stephane Bahrami, ${ }^{11,16}$ Marie-Maude Geoffray, ${ }^{14,15}$ The IDEA Study Group

To cite: Touzet S, Occelli $P$, Schröder C, et al. Impact of the Early Start Denver Model on the cognitive level of children with autism spectrum disorder: study protocol for a randomised controlled trial using a twostage Zelen design. BMJ Open 2017;7:e014730. doi:10.1136/bmjopen-2016014730

- Prepublication history for this paper is available online. To view these files please visit the journal online (http://dx.doi.org/10.1136/ bmjopen-2016-014730).

Received 13 October 2016 Revised 17 February 2017 Accepted 27 February 2017

CrossMark

For numbered affiliations see end of article.

Correspondence to Marie-Maude Geoffray; marie-maude.geoffray@ch-levinatier.fr

\section{ABSTRACT}

Introduction: Early intervention for autism spectrum disorder (ASD) in the European French-speaking countries is heterogeneous and poorly evaluated to date. Early intervention units applying the Early Start Denver Model (ESDM) for toddlers and young children with ASD have been created in France and Belgium to improve this situation. It is essential to evaluate this intervention for the political decision-making process regarding ASD interventions in European Frenchspeaking countries. We will evaluate the effectiveness of 12 hours per week ESDM intervention on the cognitive level of children with ASD, over a 2-year period.

Methods and analysis: The study will be a multicentre, randomised controlled trial, using a twostage Zelen design. Children aged 15-36 months, diagnosed with ASD and with a developmental quotient (DQ) of 30 or above on the Mullen Scale of Early Learning (MSEL) will be included. We will use a stratified minimisation randomisation at a ratio 1:2 in favour of the control group. The sample size required is 180 children (120 in the control and 60 in the intervention group). The experimental group will receive 12 hours per week ESDM by trained therapists 10 hours per week in the centre and 2 hours in the toddlers' natural environment (alternatively by the therapist and the parent). The control group will receive care available in the community. The primary outcome will be the change in cognitive level measured with the DQ of the MSEL scored at 2 years. Secondary outcomes will include change in autism symptoms, behavioural adaptation, communicative and productive language level, sensory profile and parents' quality of life. The primary analysis will use the intention-to-treat principle. An economic evaluation will be performed.

Dissemination: Findings from the study will be disseminated through peer reviewed publications and meetings.

\section{Strengths and limitations of this study}

The study population will be recruited from the general population of children with autism spectrum disorder (ASD) which strengthens the external validity of the study.

- The Early Start Denver Model (ESDM) will be conducted for 12 hours per week: 10 hours in an intervention unit and 2 hours in the toddlers' natural environment (home, nursery or preschool) encouraging generalisation of their skills.

- An innovative two-stage Zelen design will be used to avoid the disappointment of parents whose children are followed in the control group; these parents will not be informed of the ESDM intervention among other children.

- Blinding of participants will not be feasible due to the nature of the intervention; the evaluators will, however, be blinded to the intervention.

- An economic add-on evaluation will be performed to put direct and indirect costs against clinical and social outcomes.

Trial registration number: NCT02608333 (clinicaltrials.gov); Pre-results.

\section{INTRODUCTION}

\section{Background and rationale}

Autism spectrum disorder (ASD) is a heterogeneous developmental disorder with impairments in reciprocal social interaction and communication, a restricted repertoire of interests and behaviours and atypical sensory 
reactivity. ${ }^{1}$ The clinical presentation of ASD is frequently associated with intellectual disability and other developmental disorders, such as attention deficit hyperactivity disorders, and specific motor and language disorders. ${ }^{12}$ Overall ASD prevalence is about $0.7-1 \% .^{34}$ The prognosis involves individuals, families and society. ${ }^{5-7}$ Early intervention for ASD in European countries is heterogeneous and poorly evaluated to date. ${ }^{8}$ In French-speaking European countries, most children and their families have access to a public consultation centre specialised in autism. Families and children have regular consultations with a public psychologist or child and adolescent psychiatrist, but meeting schedules vary greatly (weekly, monthly or once a quarter). Moreover, children can also have speech and language therapy and/or occupational therapy and/or individual or group psychotherapy. Occupational therapy and individual or group psychotherapy are frequently based on psychoanalytic or psychodynamic traditions. ${ }^{9}$ Additional interventions may include behavioural therapies or the principles of Treatment and Education of Autistic and Related Communication Handicapped Children (TEAACH). ${ }^{10}$ Group psychotherapy is a relationship-based intervention in small groups (three to four children for two adults), generally conducted in sessions of $1 \mathrm{hr} 30 \mathrm{~min}$, twice a week. Of all interventions, speech and language therapies are those most frequently reported. ${ }^{8}$ These therapies can vary in frequency (30 min per session and up to four times per week). All these different treatments can be delivered in public or private centres. Most are refunded by social national funds and mandatory health insurance.

With regards to schooling, children have access to regular preschool with an optional special needs assistant or they may attend special preschools for children with more severe disabilities. In France, the majority of children with ASD below the age of four have $<4$ hours interventions per week and go to preschool with a special needs assistant. Educational professionals are connected with healthcare professionals.

An early comprehensive, intensive, behavioural intervention may improve the children's developmental trajectory. ${ }^{11}{ }^{12}$ Among the various early interventions, the Early Start Denver Model (ESDM) has the advantage of having been developed for young non-verbal children with ASD. ${ }^{13}$ The ESDM is a comprehensive developmental and behavioural intervention aiming to promote optimal social interactions between the child and their environment to enable the child to learn from their environment. It integrates applied behaviour analysis with developmental and relationship-based approaches. The ESDM involves a therapist who individually interacts with the child; parents or other childcare professionals (eg, nursery/preschool) can be taught ESDM techniques to use them in daily life and thereby improving generalisation of the children's skills. ESDM delivered at home 20 hours per week has demonstrated significant gains in the cognitive level of toddlers. ${ }^{14}$ The ESDM methodology is precisely detailed in a manual, including a fidelity rating scale, and rigorous training. ${ }^{13}$

Since 2011, early intervention units applying the ESDM for toddlers and young children with ASD have been created in France and Belgium to improve early intervention in French-speaking countries. In these intervention units, 12 hours per week ESDM intervention is provided through public funding. The effectiveness of 12 hours per week ESDM has yet to be demonstrated. ${ }^{12}{ }^{15}$ It is, therefore, essential to evaluate this in a population of young children with ASD in order to provide data for the political decision-making process regarding ASD interventions.

\section{Objectives}

The main objective is to evaluate effectiveness of 12 hours per week ESDM on the cognitive level in children aged 15-36 months with ASD. Cognitive level will be assessed through the developmental quotient (DQ) of the Mullen Scale of Early Learning (MSEL). The secondary objective is to measure the effectiveness on autism symptoms, behavioural adaptation, communicative and productive language levels and sensory profiles. Parents' quality of life will be also assessed. An economic evaluation will be performed to put direct and indirect costs against clinical and socioeconomic outcomes.

\section{METHODS/DESIGN}

\section{Study design}

The study will be a multicentre, randomised controlled trial, using a two-stage modified Zelen design (figure 1). ${ }^{16} 17$ The experimental group will consist of toddlers with ASD receiving 12 hours per week ESDM and the control group will consist of toddlers with ASD receiving care available in the community.

Core trial information is presented in table 1 (WHO Trial Registration Data Set).

\section{Setting}

The study will be conducted in France (five centres) and in Belgium (one centre). The centres are located in university or general hospitals. All centres have an ASD diagnostic unit and a separate ESDM unit. They receive a French-speaking population including socioeconomically disadvantaged groups.

\section{Participants}

\section{Inclusion criteria}

Children will be included if they meet the following criteria: (1) diagnosis of ASD based on the Diagnosis and Statistical Manual of Mental Disorders, Fifth Edition (DSM-5) criteria; (2) diagnosis of ASD on the Autism Diagnosis Observation Schedule (ADOS-2) ${ }^{18}$ and Autism Diagnosis Interview (ADI-R) for toddlers (we will include children above or equal to 13 , which is the research cut-off); $;^{19} \quad{ }^{20}$ (3) aged between 15 and 


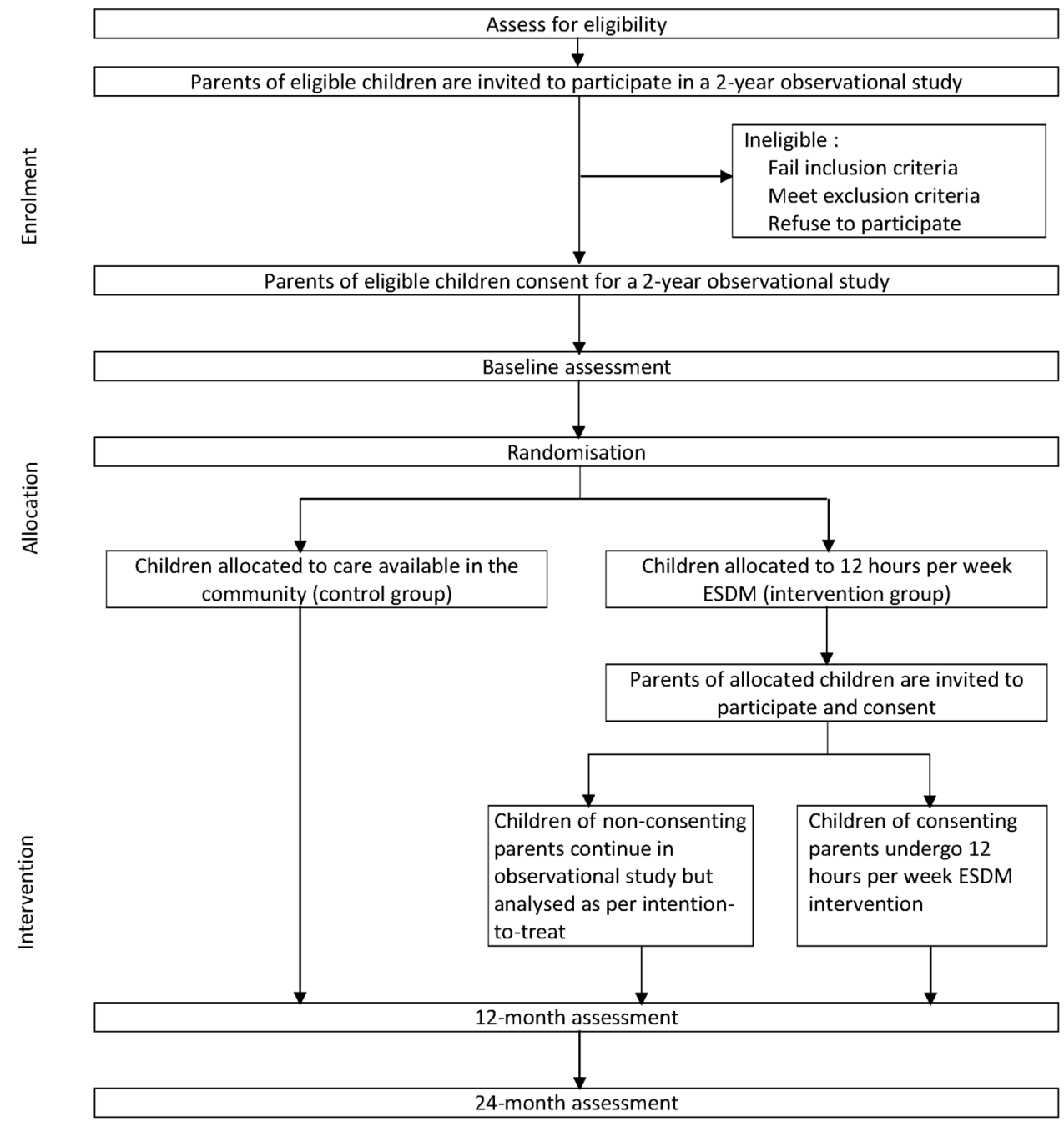

Figure 1 Flow diagram of study protocol. In the two-stage Zelen design, all parents of eligible children consent to participate in a longitudinal observational study, and in a second step, children are randomly allocated to the intervention group.

36 months; (4) DQ of 30 or above at the MSEL and (5) family living within 40 min of an ESDM unit.

\section{Exclusion criteria}

Exclusion criteria will be as follows: (1) serious neurological or physical condition, such as epilepsy requiring medication or severe sensory impairment presented by the child that would interfere with the intervention; (2) diagnosis of Rett syndrome and (3) family unavailable for a regular follow-up and intervention.

\section{Intervention conducted in the experimental group: 12 hours per week ESDM \\ Eligibility criteria for ESDM therapists}

Therapists will be speech language therapists, occupational therapists, clinical nurses and psychologists-all specialised in autism. They will all have received formal ESDM training. To ensure treatment fidelity, all therapists will have to have a score above $80 \%$ on the ESDM fidelity scale, verified once a year by an official instructor. ${ }^{21}$

\section{ESDM description}

The intervention will be provided 10 hours per week at the intervention unit and 2 hours per week in the toddlers' natural environment (home, nursery and preschool). Therapists will apply the ESDM principles outlined in the manual. ${ }^{13}$ According to the manual, 20 25 behavioural and developmental objectives will be set every 12 weeks based on observations made by therapists and parents. ESDM intervention will be modified according to the child's development as detailed in the ESDM manual.

ESDM is an individualised therapy with one therapist per child. However, according to the children's objectives, two children and two therapists can work together on social interaction. For 2 hours per week in the toddlers' natural environment, the majority of the time therapy will be delivered at home, alternatively by the therapist and the parent under the supervision of the therapist. Parents will be coached in ESDM by therapists. Therapists will give them information on their child's functioning and ESDM techniques, and guide them with 
Table 1 WHO trial registration data set

Data category
Primary registry and trial
identifying number
Date of registration in primary
registry
Secondary identifying numbers

Source of monetary or material support

\section{Information}

clinicaltrials.gov NCT02608333

11 May 2015

French institutional review board (2015-013B) sponsor (69HCL15_0278)

French Ministry of Health (Programme de Recherche sur la Performance du Système de soins)

Fondation de France (member of the Network of European Foundations for Innovative Cooperation)

\section{Primary sponsor \\ Secondary sponsor \\ Contact for public queries}

Contact for scientific queries

Public title

Scientific title

Countries of recruitment Health condition or problem studied Interventions

Key inclusion and exclusion criteria

Study type

Date of first enrolment Target sample size Recruitment Status Primary outcome Key secondary outcomes
Centre Hospitalier Le Vinatier, Bron, France

Not applicable

Marie-Maude Geoffray (PI), email: marie-maude.geoffray @ ch-le-vinatier.fr

Sandrine Touzet, email: sandrine.touzet@ chu-lyon.fr

Amelie Zelmar, email: amelie.zelmar@chu-lyon.fr

Marie-Maude Geoffray (PI), email: marie-maude.geoffray @ ch-le-vinatier.fr

Sandrine Touzet (scientific contact), email: sandrine.touzet@ chu-lyon.fr

Impact of the Early Start Denver Model (ESDM) on the cognitive level of children with autism spectrum disorder.

Impact of the ESDM on the cognitive level of children with autism spectrum disorder: study protocol for a randomised controlled trial using a two-stage Zelen design. IDEA study.

France and Belgium

Autism spectrum disorder (ASD)

Intervention: ESDM for toddlers and young children with ASD

Description: ESDM delivered by trained therapists 10 hours per week at the intervention unit and 2 hours per week in the toddlers' natural environment (home, nursery, preschool). Control: care available in the community, i. e. consultations with a psychologist or child and adolescent psychiatrist, speech and language therapy, occupational therapy, individual or group psychotherapy.

Age: between 15 and 36 months

Sex: male or female

Inclusion criteria: diagnosis of ASD (Diagnosis and Statistical Manual of Mental Disorders, Fifth Edition, and Autism Diagnosis Observation Schedule), DQ of 30 or above at the MSEL, family living within 40 min of an ESDM unit. Exclusion criteria: serious neurological or physical condition, diagnosis of Rett syndrome, family unavailable for a regular follow-up and intervention.

Interventional

Allocation: randomised 1:2; parallel assignment; blinding: assessor blind; two-stage modified Zelen design

NA

180

NA

Change in developmental quotient (DQ) measured using the MSEL, scored at 24 months Child development, autism symptoms, quality of life of parents, healthcare resource use. positive feedback to practice ESDM with their child. Moreover, parents will be encouraged to use ESDM in daily activities and for periods of special ESDM time (around $30 \mathrm{~min}$ per day in addition to the 2 hours weekly therapy). Moreover, the therapist will convey information about autism and ESDM techniques and share the child's developmental objectives with childcare professionals of the children's nurseries and preschools.

At least once a month, parents will have an additional consultation with the referent child psychologist or psychiatrist in order to assist the family further in the understanding of ASD, coping and to reinforce support for parents and siblings. Four family workshops (2 hours each) per year will be proposed to parents by professionals in order to share general information about autism, rights and social aids and to solve problems related to challenging behaviour, food selectivity and disorders, together with other parents of children with ASD.

Strategies to improve adherence to ESDM interventions

At least once a month, the referral child psychologist or psychiatrist will contact the parents in order to accompany 
the family and reinforce the motivation of the parents so that their child attends each ESDM session.

\section{Interventions that are permitted during the trial}

Parents will be free to seek other care available in the community.

\section{Control group}

Parents with toddlers allocated to the control group will be informed and referred to care available in the community. Psychiatrists and psychologists of ASD diagnostic units will guide parents toward interventions such as weekly speech and language therapy, occupational therapy as well as individual or group psychotherapy. All different treatment approaches will be quantified (type, frequency, intensity and duration) and taken into account in comparative analyses.

\section{Allocation sequence generation and randomisation}

All eligible toddlers, whose parents have provided consent for them to participate, will be randomised using stratified minimisation at a ratio 1:2 in favour of the control group (one toddler assigned to the intervention group for two toddlers assigned to the control group). A random element will be used, so that children will be assigned to the treatment arm which will minimise the imbalance with $90 \%$ probability. The minimisation factors will be the centre, the children's age, the DQ score on the MSEL and the severity of the ADOS score at inclusion. Randomisation will be performed using a computer program generated in the statistical software SAS by the centre for clinical research of the university teaching hospital of Lyon (Hospices Civils de Lyon, France). The algorithm will be held and controlled centrally by an independent statistician. Details of stratified minimisation will be provided in a separate document unavailable to those who enrol toddlers.

Once informed of group allocation, trial administrators will inform the lead investigator of the centre, who will plan ESDM intervention delivery if applicable, or simple follow-up.

\section{Child assessment}

All children will be evaluated in an ASD diagnostic unit of the participating centres. The diagnosis of ASD will be confirmed by expert child psychiatrists at the time of inclusion. Children of both groups will benefit from early evaluation and will have the same follow-up evaluations over the 2-year study period in the diagnostic units.

\section{Blinding}

Owing to the nature of the intervention, parents of the children or ESDM therapists cannot be blind to the allocation group. ESDM therapists will not be involved in the diagnosis. The MSEL and other instruments will be administered by an assessor blind to the allocation group. At assessment points, children, parents and schools will be reminded that the assessor is blind to treatment allocation and that anything related to this should not be discussed with them. Blinding of outcome assessors and data analysts will be fully achieved.

\section{Outcome measures and tools}

All outcomes will be assessed at baseline, 12 and 24 months of follow-up (figure 2). Additionally, demographic and socioeconomic data will be obtained at the same times points for descriptive purposes.

The primary outcome will be the change in DQ measured using the MSEL, scored from baseline to 24 months. Secondary outcome measures will focus on child development and autism symptoms, quality of life of parents and healthcare resource use.

\section{To assess child cognitive level}

- The MSEL is a direct observation tool measuring cognitive levels for children from birth to 68 months. ${ }^{22}$ It is a reliable, validated test widely used to evaluate the paediatric ASD population. ${ }^{23} 24$ As most of the children are likely to score too low to use the Early Learning Composite Score of the MSEL manual, we will calculate the DQ score (ie, developmental age equivalents divided by chronological age). As the Early Learning Composite Score, the DQ score will be obtained from four subscales (fine motor, visual reception, expressive language and receptive language). DQ is usually used for children with intellectual disabilities or developmental delays. ${ }^{23} 25-27$ The MSEL has been translated and backtranslated in French for the purpose of the study.

\section{To assess child autism symptoms and change in autism symptoms}

- The ADOS-2 is a semistructured standardised observation tool, which measures core autism symptoms, that is to say reciprocal sociocommunicative interactions, repetitive and restrictive behaviours. ${ }^{18}$ A score in social affect and restrictive repetitive behaviours is measured, and an overall autism severity score will be calculated. ${ }^{28}$

- The Brief Observation of Social Communication Change (BOSCC) instrument measures social changes in children with ASD. ${ }^{29}$ Social changes are observed using standardised activities protocol between therapist and child. The instrument is scored from videos.

\section{To assess child behavioural adaptation}

- The Vineland Adaptive Behaviour Scales second version (VABS-2) measures personal and social skills needed for everyday living. ${ }^{30}$ It assesses socialisation, communication, motor and daily living skills, based on parent interviews. Standard scores for each of the four studied domains are provided and a composite standard score is derived from the four domains.

\section{To assess child communicative and productive language} level

- The Communication and Symbolic Behaviour Scales Developmental Profile (CSBS-DP) is a self-administered 


\begin{tabular}{|c|c|c|c|c|}
\hline Time point & Enrolment & $\begin{array}{l}\text { Baseline } \\
\text { (year 0) }\end{array}$ & $\begin{array}{c}12 \text { month } \\
\text { assessment }\end{array}$ & $\begin{array}{l}24 \text { month } \\
\text { assessment }\end{array}$ \\
\hline \multicolumn{5}{|l|}{ Enrolment } \\
\hline \multicolumn{5}{|l|}{ Eligibility screen } \\
\hline Diagnosis and Statistical Manual of Mental Disorders, Fifth Edition (DSM-V) criteria & $x$ & & & \\
\hline Autism Diagnosis Observation Schedule (ADOS-2) & $x$ & & & \\
\hline Autism Diagnosis Interview (ADI-R) for toddlers & $\mathrm{x}$ & & & \\
\hline Informed consent & $\mathrm{x}$ & & & \\
\hline Allocation & & $\mathrm{x}$ & & \\
\hline \multicolumn{5}{|l|}{ Intervention } \\
\hline \multicolumn{5}{|l|}{12 hours per week ESDM intervention } \\
\hline \multicolumn{5}{|l|}{ Care available in the community } \\
\hline \multicolumn{5}{|l|}{ Assessments } \\
\hline Demographic data & & $\mathrm{X}$ & & \\
\hline Socio-economic data & & $\mathrm{x}$ & $\mathrm{x}$ & $x$ \\
\hline \multicolumn{5}{|l|}{ To assess child cognitive level } \\
\hline Mullen Scale of Early Learning (MSEL) (primary outcome) & & $\mathrm{x}$ & $\mathrm{x}$ & $\mathrm{x}$ \\
\hline \multicolumn{5}{|l|}{ To assess child autism symptoms and change in autism symptoms } \\
\hline Autism Diagnosis Observation Schedule (ADOS-2) & & $\mathrm{x}$ & $\mathrm{x}$ & $x$ \\
\hline Brief Observation of Social Communication Change (BOSCC) & & $\mathrm{X}$ & $\mathrm{x}$ & $\mathrm{x}$ \\
\hline \multicolumn{5}{|l|}{ To assess child behavioural adaptation } \\
\hline Vineland Adaptive Behaviour Scales second version (VABS-2) & & $\mathrm{x}$ & $\mathrm{x}$ & $x$ \\
\hline Communication and Symbolic Behaviour Scale Developmental Profile (CSBS-DP) & & $\mathrm{x}$ & $\mathrm{x}$ & $\mathrm{x}$ \\
\hline Dyadic Communication Measure for Autism (DCMA) & & $\mathrm{X}$ & $\mathrm{x}$ & $x$ \\
\hline Development of expressive language & & $\mathrm{x}$ & $\mathrm{x}$ & $x$ \\
\hline \multicolumn{5}{|l|}{ To assess child sensory reactivity } \\
\hline Dunn's Sensory Profile for toddlers & & $\mathrm{x}$ & $x$ & $x$ \\
\hline \multicolumn{5}{|l|}{ To assess the parents burden and quality of life } \\
\hline The CareQuol-7D & & $\mathrm{x}$ & $\mathrm{x}$ & $x$ \\
\hline
\end{tabular}

Figure 2 Schedule of enrolment, interventions and assessments (SPIRIT template).

parent report about social communication, expressive speech/language and symbolic functioning. It provides an overall score. ${ }^{31}$

- The Dyadic Communication Measure for Autism (DCMA) ${ }^{32-34}$ is a direct observation instrument of the communication between a parent and a child with autism. It rates parental and child mutual shared attention, child communication (initiation and response) and parental communication style (synchronous/asynchronous). Coding is done based on a video of the parent and child playing together.

- The 'development of expressive language' is a standardised French scale (Development du Language de Production en Français-DLPF) measuring the development of expressive language in French, based on a selfadministered parent report. ${ }^{35}$ It was derived from The MacArthur-Bates Communicative Development Inventories. ${ }^{36}$ The level four of the DLPF will be administered at each assessment. The score obtained is the number of words and length of sentences.

\section{Assessment sensory reactivity}

- The Dunn's Sensory Profile for toddlers from 7 to 36 months determines how children process sensory information in everyday situations. ${ }^{37}$ The six sensory system scores (auditory, visual, touch, movement, body position and oral expression) and the four sensory pattern scores (seeking, avoiding, sensitivity and registration) will be used. It is a self-administered parent report.

To assess the parent burden and quality of life

- The CareQuol-7D is a validated instrument to measure the impact on the carer's quality of life, that is, the parents. ${ }^{38}$ It is a self-administered parent report that provides an overall score.

\section{Intervention process measures}

Irrespective of group assignment, parents may look for other care available in the community (additional speech language therapy, occupational therapy, interventions by educators or psychologists providing behavioural analysis, etc). In both groups, type, frequency, intensity and duration of interventions will be collected. Moreover, in the experimental group, adherence to the ESDM will be monitored with the fidelity scale of the published manual. ${ }^{13}$

\section{Economic evaluation}

An economic evaluation will be performed alongside the clinical trial to put direct and indirect costs related to interventions against clinical and social outcomes. The evaluation will be conducted from a general societal perspective, encompassing perspectives of the national health and social care systems, as well as patients. 
A cost-consequences framework will be used to accommodate all relevant perspectives and outcomes. Time horizon will be of two years for the primary analysis, in line with study duration. Healthcare, social and personal resources devoted to childcare (day care and medical) will be measured throughout the study. ${ }^{39}$ Patient day care organisation, school attendance and medical and social care interventions during the preceding year will be recorded during each study visit (baseline, 12 months and 24 months). National unit costs for care and interventions will be applied to estimate direct costs.

Parental employment changes related to ASD will be recorded and valued using the human capital approach. Analysis of socioeconomic data will be adapted to trial results and to the nature and diversity of toddlers' care in the control group, which are unknown to date, and will therefore be exploratory in nature. The costconsequences framework will allow concomitant publication of all relevant costs and outcomes, avoiding overemphasis on any of them. ${ }^{40}$

\section{Participant enrolment}

Toddlers will be enrolled by psychiatrists of the centres. The number of toddlers usually in care in the participating centres should be sufficient for the recruitment required. Nevertheless, in order to facilitate inclusions, healthcare professionals, usually meeting with children with ASD (paediatricians, speech language therapists and occupational therapists), will be contacted by postal mail to inform them of the study, or informed by specific meetings.

\section{Informed consent according to the two-stage Zelen design}

When toddlers meet the criteria for participation, parents will be informed by an expert child psychiatrist about the 24-month observational study, including an early evaluation and two evaluations over the follow-up period. They will be asked to sign written informed consent. Children will be subsequently randomised into intervention or control groups. Only the parents of children randomised to the intervention group will then be asked if they are willing to participate in an additional study involving a 12 hours per week ESDM intervention over the 24-month period. If they agree, they will be asked to sign a second written informed consent for the ESDM intervention. The parents of the children allocated to the control group will not be told about this further study or about the intervention group, but their child will be followed up as agreed initially (baseline evaluation and two yearly follow-up evaluations). Parents with children allocated to the ESDM intervention group may refuse to consent to the intervention, as it is demanding. In which case, children will remain in the study for assessment, as agreed initially. We believe this 'crossover' will have an acceptable probability $(<10 \%)$. $^{41-43}$

\section{Sample size}

The primary outcome is the DQ measured using the MSEL at 24 months after randomisation. Given the limited number of 12 hours per week ESDM interventions available at the participating centres, an unequal randomisation with a ratio 1:2 (experimental: control groups, respectively) will be used.

At 24 months after the randomisation, a mean difference of at least 15 points in the $\mathrm{DQ}(\mathrm{SD}=25)$ is expected between the groups. This hypothesis was based on the results of two studies. One of theses studies aimed to evaluate an ESDM intervention where children received 15-20 hours group-based and 1 hour with one therapist for one child. The effect size found was of 10 points for the DQ. ${ }^{44}$ The other study was a pilot study conducted in the principal investigator's (PI) centre, where children received ESDM 12 hours per week with one therapist for one child, over 9 months, which found an improvement of $11 \mathrm{DQ}$ points at the end of the study. Assuming that the 12 hours per week ESDM with one therapist for one child will be more intense than the group-based intervention, and carried out over a longer period than the pilot study, we expect a greater improvement in DQ. However, we do not expect the same improvement the second year of intervention because it is reported that children gain most of the improvement during the first year. ${ }^{14}$

To detect such a difference with a two-sided 5\% significance and a power of $90 \%$, we calculate that we will need to recruit 132 children (44 in the intervention and 88 in the control group). The required sample size has to be inflated to accommodate for the Zelen design and its dilution bias, and for attrition and dropout during the study period. Assuming a $10 \%$ non-consent rate for children randomised to the intervention group increases the sample size by $1 /(1-0.10)^{2}=20 \%,{ }^{45}$ and allowing a dropout rate of $10 \%$, according to the previous randomised controlled trial, ${ }^{14}$ will increase the total sample size to 180 children (120 in the control and 60 in the intervention group - see table 2).

\section{Data collection and management}

The study data will be collected on a secure electronic case report form (eCRF) that will be available at each centre through an internet portal. No personal identifying information will be mentioned on the eCRF. Each subject included in the study will be assigned a unique identification number that will consist of the identification number of the investigational centre, the initials of the patient and the chronological inclusion number of the patient.

Multiple external validation checks will be applied: examination of the source documents and crosschecking with the data recorded in the eCRF as to its accuracy, the presence of missing data and the consistency of data. The eCRF will only include the data necessary for the analysis to be reported in a scientific publication.

All study data will be stored securely in the University Hospital of Lyon. All electronic data will be secured on 
Table 2 The steps involved in calculating the sample size of the study

\begin{tabular}{|c|c|c|c|c|}
\hline Step & Hypothesis & $\begin{array}{l}\text { Total number of } \\
\text { patients to be } \\
\text { included }\end{array}$ & $\begin{array}{l}\text { Total number of } \\
\text { patients to be } \\
\text { included in the } \\
\text { intervention group }\end{array}$ & $\begin{array}{l}\text { Total number of } \\
\text { patients to be } \\
\text { included in the } \\
\text { control group }\end{array}$ \\
\hline$\# 1$ & $\begin{array}{l}\text { To detect a difference of at least } 15 \text { points in the } \mathrm{DQ} \\
(\mathrm{SD}=25) \text { with a two-sided } 5 \% \text { significance and a } \\
\text { power of } 90 \% \text { with a ratio } 1: 2 \text { (intervention:control) }\end{array}$ & 132 & 44 & 88 \\
\hline \#2 & $\begin{array}{l}\text { A } 10 \% \text { cross-over rate in the control group involve a } \\
20 \% \text { dilution rate }\end{array}$ & $132+27=159$ & $44+9=53$ & $88+18=106$ \\
\hline \#3 & A $10 \%$ drop-out rate over the 24-month follow-up & $159+21=180$ & $53+7=60$ & $106+14=120$ \\
\hline
\end{tabular}

a password-protected laptop. Paper-based study documents will be stored in a secure filing cabinet at each centre. All electronic documents containing names or personal identifying information, necessary for the follow-up of the study, will be stored separately from other study data and protected by a code number. Access to these files will be limited to research staff involved in the study.

The statistician for the final analysis will receive checked and validated data from the eCRF with no personal identifying information.

There are no current plans for granting public access to the full protocol, participant-level data set or statistical code. However, if researchers wish to access the data set (eg, for conduct of secondary analysis or meta-analysis) the project management committee will facilitate this.

\section{Statistical considerations}

Owing to the Zelen design and its dilution bias, the analysis will be undertaken using the intention-to-treat principle. All randomised patients will be analysed according to their allocation group, regardless of whether they will start or complete the intervention protocol. Patients who will be randomised to the intervention but who refuse their allocated treatment and who opt for usual care (control group) will be analysed as though they had received the intervention. The analysis by intention-to-treat will maintain the baseline comparability of the groups.

There is no interim analysis planned. Demographic and clinical characteristics, as well as baseline data, will be presented to assess the baseline comparability of the two groups. The comparability will be verified on the main characteristics using the Student's t-test or, when appropriate, the Wilcoxon-Mann-Whitney rank test for continuous variables and the $\chi^{2}$ test or the exact Fisher test if the conditions of application of the $\chi^{2}$ test were not fulfilled for the qualitative variables.
Concerning the analysis of the primary outcome, descriptive statistics will be presented for each group as the mean change (SD, 95\% CIs) in DQ score from baseline to 24 months after randomisation. The longitudinal association between ESDM intervention and DQ scores over the 24-month study period will be estimated using a linear mixed-effects regression model. ${ }^{46}$ Mixed models have the advantage of dealing with missing values because they use all of the available data from a patient over the study period. The $\mathrm{DQ}$ score at each follow-up visit will be defined as the dependent variable. The first model will include the following as independent variables: study group, time. The group will be coded as a dichotomous variable. Time will be treated as a continuous variable to account for the variability of the point in time at which the measurements are obtained. The intercept and slope for time will be specified as random effects to account for the initial difference and change difference over time between individuals. An interaction effect between intervention and time will be investigated to test if the slope of change is different between intervention and control groups. A repeated statement will be added to model the best covariance structure of the within-patient error, according to the Akaike's information criterion. This basic model will be adjusted for all minimisation variables (centre age at diagnosis, DQ score at baseline and autism severity score on the ADOS-2 scale). Adjusted models will additionally control for other patient characteristics, family characteristics (social class based on occupation and education level) and the total number of ESDM hours toddlers will have received over the study period. Additional covariates can be included if they are significant at 0.05 level according to the likelihood ratio test or if they alter the coefficient of the intervention effect $>10 \%$, when removed from the analysis. All models will be performed with the SAS MIXED procedure.

Model assumptions will be verified according to residual analysis. If most of the assumptions are not met, 
other alternatives such as transformation for DQ score or generalised linear mixed model will be examined.

For the secondary outcomes, change in other scores from baseline to 24 months after randomisation will be analysed in the same way as the primary outcome.

All tests will be two sided and carried out at the 5\% level of significance. Statistical analyses will be performed using SAS 9.3 (SAS Institute, Cary, North Carolina, USA) by the centre for clinical research of the teaching hospital of Lyon (Hospices Civils de Lyon, France).

\section{Organisation}

The Trial Steering Committee (TSC) will be responsible for overseeing the progress of the trial and will meet at regular intervals. The TSC includes the PI, the investigators of the centres and the trial coordinators. The TSC has developed the study protocol and is responsible for data collection, management, publications and the final data set. The committee is responsible for finding solutions to unforeseen questions/problems that may arise in the course of the study.

According to French law, the study does not require a formal data monitoring committee as it is a trial with known minimal risks.

\section{Dissemination}

The study team will be committed to full disclosure of the results of the trial. The results of the study will be disseminated at several national and international meetings, and as articles published in national and international peer-reviewed journals. The study will be implemented and reported in line with the CONSORT statement. Each paper or abstract will be submitted to the appropriate subcommittee for review of its appropriateness and scientific merit prior to submission. The study team will adhere to defined authorship criteria as per the International Committee of Medical Journal Editors.

\section{DISCUSSION}

\section{The 12 hours per week ESDM intervention}

We expect that our strategy of 10 hours of ESDM at the intervention unit and 2 hours in the toddlers' natural environment (home, nursery or preschool) will encourage use of the ESDM by parents at home and in natural environments of daily life and thus reinforce the intervention.

The study will be the first randomised multicentre controlled trial investigating the effectiveness of a behavioural and developmental intervention such as ESDM in a French-speaking European population.

The present study will be a randomised clinical trial with a large sample size of very young children with ASD receiving a therapist-ESDM semi-intensive intervention during 2 years. The inclusion criteria are wide enough to take into account the heterogeneity of ASD at this age and will not exclude children with genetic disorders associated with ASD (with the exception of Rett syndrome) as in other studies. ${ }^{32} 4748$ Moreover, this study will address needs of non-verbal toddlers for whom few studies are available. ${ }^{12} 3248-50$

\section{The two-stage Zelen design}

Contrary to the classic randomisation procedure for clinical trials, the Zelen design proposes that randomisation is performed before patients give their consent to participate. More specifically, in the two-stage Zelen design, all parents of eligible children consent to participate in a longitudinal observational study, and second, children are randomly allocated to the intervention group. Only the parents of these children are informed about the intervention, and give their written consent for it. This design presents some significant advantages. First, it should avoid a strong feeling of disappointment among parents, and reduce the risk of study withdrawal if their child is not randomised to the intervention group, as 12 hours per week ESDM is highly desired by families. ${ }^{43}$ Thus, it may promote participation and limit withdrawal of consent. ${ }^{42}$ Furthermore, the use of the Zelen design will minimise the bias typically encountered in traditionally designed randomised clinical trials where participant expectations may influence study outcomes, that is, parents' expectation for their child and their participation in the behavioural intervention.

\section{An effectiveness study}

As the aim of this study is to evaluate the 12 hours per week ESDM strategy, compared with the care available in the community within a broad, heterogeneous and representative population, we will conduct an effectiveness study. ${ }^{51}$ The control group of this study will receive heterogeneous interventions available in the community with different intensity and practices. ${ }^{8}$

In the intervention group, we will notify to what extent the participants are compliant with the ESDM intervention.

\section{The outcome measures}

Because the needs of children with ASD are complex, a diversity of outcome measurement tools is used to collect evidence about the child's progress. ${ }^{52}$ Their cognitive abilities play a central role in the manifestation of core and associated symptoms in ASD. ${ }^{23}$ The MSEL is commonly used as a measure of cognitive and language skills in ASD. The MSEL covers the range of age and skills differences (eg, verbal or non-verbal children). It has been used in previous studies and allows comparison with other papers. ${ }^{14}$

\section{Author affiliations}

${ }^{1}$ Pôle Information Médicale Evaluation Recherche, Hospices Civils de Lyon, Lyon F-69003, France

${ }^{2}$ Laboratoire Health Services and Performance Research, EA 7425 HESPER, Université de Lyon, Lyon F-69008 France 
${ }^{3}$ Department of Child and Adolescent Psychiatry, Hopitaux universitaires de Strasbourg, Strasbourg F-67000, France

${ }^{4}$ CNRS UPR 3212-Team 9, Strasbourg University, Strasbourg F-67000, France

${ }^{5}$ Centre Hospitalier Saint Jean de Dieu, Lyon F-69008, France

${ }^{6}$ Pôle Universitaire de Psychiatrie de l'Enfant et de l'Adolescent, Centre Hospitalier Spécialisé Henri Laborit, Saint Benoît F-86280, France

${ }^{7}$ Child and Adolescent Psychiatry Department, Unité de Recherche Clinique, Université de Poitiers, Poitiers F-86000, France

${ }^{8}$ Hôpital Universitaire des Enfants Reine Fabiola, Université Libre de Bruxelles, Bruxelles 1020, Belgium

${ }^{9}$ Office Médico-Pédagogique, University of Geneva, Geneva, Switzerland

${ }^{10}$ Service Universitaire de Psychiatrie de l'Enfant et de l'Adolescent, Centre Hospitalier de Versailles, Le Chesnay F-78150, France

${ }^{11}$ EA 4047 HANDIReSP, Université de Versailles Saint-Quentin-en-Yvelines, Versailles F-78000, France

${ }^{12}$ Université Paris-Sud, CESP, INSERM, UVSQ, Université Paris-Saclay, U1178, Maison de Solenn, Paris cedex 14, France

${ }^{13}$ Department of Public Health, AP-HP, Hôpital Paul Brousse, Villejuif F-94800, France

${ }^{14}$ Department of child and adolescent psychiatry, Centre Hospitalier le Vinatiers, Bron F-69500, France

${ }^{15}$ Université de Lyon, Lyon F-69008, France

${ }^{16}$ CIC 1429, INSERM, AP-HP, Hôpital Raymond-Poincare, Garches F-92380, France

Acknowledgements The authors would like to thank Sally Rogers, Elizabeth Fulton, Cynthia Zermut and Marie Rocha for their training in ESDM. They are grateful to Catherine Lord and Rebecca Grzadzinski for use of the BOSCC. Particular acknowledgements are addressed to Jonathan Green for his expert advice and to Catherine Aldred for her training in DCMA methodology. They thank all teams of the different study sites for their ongoing support of this project. Thanks also to Paul Belhouchat, a member of the Centre for Autism Resources of Lyon (Rhône-Alpes) who provided references. The authors are grateful to Philip Robinson for his help with manuscript preparation and Isabelle Bluche, Translator-Editor, Translands Traductions, for editing this paper

Collaborators Lyon-CH du Vinatier: MMG, NG, Stéphane Grisi, Stéphanie Marignier, Jean-Marc Useo, Alain Pourrat, Chloé Peter, Flavia Mengarelli, Natacha Gallifet, Fanny Rumillat, Gaelle Tenant;

Lyon-CH Saint Jean de Dieu: SM, Sandrine Sonié, Marc Zimmerman, Valérie Malo, Céline Grosmaître Jacob;

Versailles-Paris: Stéphane Bahrami, BF, MJO, MS, Camille Queste;

Poitiers: LG, Florence Raffeneau, Sophie Gilet, Mélanie Ammeloot;

Strasbourg: Anne Danion-Grilliat, Emilie Florence, CS, Valérie Vecchionacci, Lucie Janssen, Anna Dubrovskaya, Monique Rohmer, Bénédicte Lambs

Bruxelles: Véronique Delvenne, RS, Sophie Carlier, Lesley Ducenne;

Genève: MS

Lyon—HCL: Cyrille Colin, AD, PO, ST, AZ, Laeticia Bouveret.

Contributors ST, MMG, SB and PO conceived and designed the project, and $M M-G$ is leading the coordinator of the trial. ST, MMG and PO drafted the protocol and procured the project funding. MMG is responsible for study implementation, staff training and supervision. SB and MMG designed and are leading the economic evaluation. ST and AD contributed to the sample size calculation, the randomisation procedure and the statistical plan, and are responsible for data management, randomisation and statistical analysis. BF gave advice to the randomisation procedure. MMG, SM, CS, MSp, RS, NG, MJO and MSc are responsible for recruitment and evaluation of children. All authors critically reviewed and approved the final version of the manuscript.

Funding This study is supported by a grant from the Programme de Recherche sur la Performance du Système de soins (PREPS 14-0533) from the French Ministry of Health (Ministère chargé de la Santé, Direction de l'Hospitalisation et de l'Organisation des Soins) and a grant from the Fondation de France, a member of the Network of European Foundations for Innovative Cooperation.
Disclaimer The funders and sponsor have no role in study design, data collection, management, data analysis and interpretation of data, in the writing of the report or in the decision to submit the manuscript for publication.

Competing interests None declared.

Patient consent Obtained.

Ethics approval The ethical issues raised by Zelen design were discussed by the Ethics Committee of the university teaching hospital of Lyon; the study appeared to protect the children and the Zelen design was an ethically acceptable methodological alternative for this behavioural trial, and was therefore given approval. Ethics approval was obtained from the French Institutional Review Board (reference No 2015-013 B-"Comité de Protection de Personnes") and the French Data Protection Agency (reference DR-2015-319-“Commission Nationale Informatique et Liberté"). Ethics approval was also obtained from the Belgium Institutional Review Board (reference P2016/554, reference CCB B406201630678- “Comité d'Ethique Erasme-ULB").

Amendments will be reported to relevant regulatory parties.

Provenance and peer review Not commissioned; externally peer reviewed.

Data sharing statement The trial statisticians will have access to the data set for the analysis of trial outcomes. The PI will have access to the data and will take full responsibility for the analysis and publication of the results. Once the main analyses have been undertaken, data will be available to principal and other investigators subject to approval of data analysis plans by the steering committee.

Open Access This is an Open Access article distributed in accordance with the Creative Commons Attribution Non Commercial (CC BY-NC 4.0) license, which permits others to distribute, remix, adapt, build upon this work noncommercially, and license their derivative works on different terms, provided the original work is properly cited and the use is non-commercial. See: http:// creativecommons.org/licenses/by-nc/4.0/

\section{REFERENCES}

1. American Psychiatric Association. Diagnostic and statistical manual of mental disorders. 5th edn. Arlington, VA: American Psychiatric Publishing, 2013.

2. Charman $T$, Pickles $A$, Simonoff $E$, et al. IQ in children with autism spectrum disorders: data from the Special Needs and Autism Project (SNAP). Psychol Med 2011;41:619-27.

3. Yeargin-Allsopp M. The prevalence and characteristics of autism spectrum disorders in the ALSPAC cohort. Dev Med Child Neurol 2008;50:646.

4. Fombonne $\mathrm{E}$. The prevalence of autism. JAMA 2003:289:87-9.

5. Howlin P. Living with impairment: the effects on children of having an autistic sibling. Child Care Health Dev 1988;14:395-408.

6. Hayes SA, Watson SL. The impact of parenting stress: a meta-analysis of studies comparing the experience of parenting stress in parents of children with and without autism spectrum disorder. J Autism Dev Disord 2013:43:629-42.

7. Ganz ML. The lifetime distribution of the incremental societal costs of autism. Arch Pediatr Adolesc Med 2007;161:343-9.

8. Salomone E, Beranová Š, Bonnet-Brilhault F, et al. Use of early intervention for young children with autism spectrum disorder across Europe. Autism 2016;20:233-49.

9. Evans B. How autism became autism: The radical transformation of a central concept of child development in Britain. Hist Human Sci 2013;26:3-31.

10. Schopler E, Mesibov GB, Hearsey K. Structured teaching in the TEACCH system,1995. In: Schopler E, Mesibov GB eds. Learning and cognition in autism. New York: Kluwer Academic/Plenum, 243--267

11. Warren Z, McPheeters ML, Sathe N, et al. A systematic review of early intensive intervention for autism spectrum disorders. Pediatrics 2011;127:e1303-11.

12. Maglione MA, Gans D, Das L, et al. Technical expert panel. Nonmedical interventions for children with ASD: recommended guidelines and further research needs. Pediatrics 2012;130(Suppl 2):S169-78.

13. Rogers S, Dawson G. Early Start Denver Model for young children with Autism: promoting language, learning, and engagement. Traduit par Rogé. Edition Dunod, Paris: Guilford Press, 2013. 
14. Dawson G, Rogers S, Munson J, et al. Randomized, controlled trial of an intervention for toddlers with autism: the Early Start Denver Model. Pediatrics 2010;125:e17-23.

15. Fava L, Strauss K. Response to early intensive behavioral intervention for autism--an umbrella approach to issues critical to treatment individualization. Int J Dev Neurosci 2014;39:49-58.

16. Torgerson DJ, Roland M. What is Zelen's design?. BMJ 1998;316:606

17. Campbell R, Peters T, Grant C, et al. Adapting the randomized consent (Zelen) design for trials of behavioural interventions for chronic disease: feasibility study. J Health Serv Res Policy 2005;10:220-5.

18. Lord C, Rutter M, DiLavore PC, et al. Autism diagnostic observation schedule. Los Angeles, CA: Western Psychological Services, 2012.

19. Lord C, Rutter M, Le Couteur A. Autism diagnostic interview-revised: a revised version of a diagnostic interview for caregivers of individuals with possible pervasive developmental disorders. J Autism Dev Disord 1994;24:659-85.

20. Kim SH, Thurm A, Shumway $\mathrm{S}$, et al. Multisite study of new autism diagnostic interview-revised (ADI-R) algorithms for toddlers and young preschoolers. J Autism Dev Disord 2013;43:1527-38.

21. McConachie $\mathrm{H}$, Fletcher-Watson S, Working group 4, COST action 'enhancing the scientific study of early autism'. Building capacity for rigorous controlled trials in autism: the importance of measuring treatment adherence. Child Care Health Dev 2015;41:169-77.

22. Mullen EM. Mullen scales of early learning. AGS Edition. Circle Pines, MN: American Guidance Service, 1995.

23. Bishop SL, Guthrie W, Coffing M, et al. Convergent validity of the mullen scales of early learning and the differential ability scales in children with autism spectrum disorders. Am J Intellect Dev Disabil 2011:116:331-43.

24. Swineford LB, Guthrie W, Thurm A. Convergent and divergent validity of the Mullen Scales of early learning in young children with and without autism spectrum disorder. Psychol Assess 2015;27:1364-78.

25. Lord C, Risi S, DiLavore PS, et al. Autism from 2 to 9 years of age. Arch Gen Psychiatry 2006;63:694-701.

26. Pickles A, Anderson DK, Lord C. Heterogeneity and plasticity in the development of language: a 17-year follow-up of children referred early for possible autism. J Child Psychol Psychiatry 2014;55:1354-62.

27. Rogers SJ, Estes A, Lord C, et al. Effects of a brief Early Start Denver model (ESDM)-based parent intervention on toddlers at risk for autism spectrum disorders: a randomized controlled trial. J Am Acad Child Adolesc Psychiatry 2012;51:1052-65.

28. Gotham K, Risi S, Pickles A, et al. The Autism Diagnostic Observation Schedule: revised algorithms for improved diagnostic validity. J Autism Dev Disord 2007;37:613-27.

29. Grzadzinski R, Carr T, Colombi C, et al. Measuring changes in social communication behaviors: preliminary development of the Brief Observation of Social Communication Change (BOSCC). J Autism Dev Disord 2016;46:2464-79.

30. Sparrow S, Cicchetti DV, Balla DA. Vineland adaptive behavior scales. 2nd edn. VinelandTM-II: Pearson, 2005.

31. Wetherby AM, Allen L, Cleary J, et al. Validity and reliability of the communication and symbolic behavior scales developmental profile with very young children. J Speech Lang Hear Res 2002;45:1202-18.

32. Green J, Charman T, McConachie H, et al. Parent-mediated communication-focused treatment in children with autism (PACT): a randomised controlled trial. Lancet 2010;375:2152-60.
33. Aldred C, Green J, Emsley R, et al. Brief report: mediation of treatment effect in a communication intervention for pre-school children with autism. J Autism Dev Disord 2012;42:447-54.

34. Pickles A, Harris V, Green J, et al. Treatment mechanism in the MRC preschool autism communication trial: implications for study design and parent-focussed therapy for children. J Child Psychol Psychiatry 2015;56:162-70.

35. Bassano D, Labrell F, Champaud C, et al. Le DLPF : Un nouvel outil pour l'évaluation du développement du langage de production en français. Enfance, $\mathrm{n}^{\circ}$ 2. Presses universitaires de France. 2005 http://www.umr7023.cnrs.fr/o-Le-DLPF-un-nouvel-outil-pour-I.htm

36. Fenson L, Dale PS, Reznick JS, et al. MacArthur communicative development inventory: users guide and technical manual. San Diego, CA: Singular, 1993.

37. Dunn W. Sensory profile 2 user's manual. San Antonio, TX: Psychological Corporation, 2014

38. Hoefman R, Payakachat N, van Exel J, et al. Caring for a child with autism spectrum disorder and parents' quality of life: application of the CarerQol. J Autism Dev Disord 2014;44:1933-45.

39. Byford S, Cary M, Barrett B, et al. Cost-effectiveness analysis of a communication-focused therapy for pre-school children with autism: results from a randomised controlled trial. BMC Psychiatry 2015;21:316.

40. Glick HA, Doshi JA, Sonnad SS, et al. Economic evaluation in clinical trials. 2nd edn. Oxford: University Press, 2015.

41. Schellings R, Kessels AG, ter Riet G, et al. The Zelen design May be the best choice for a heroin-provision experiment. J Clin Epidemiol 1999;52:503-7.

42. Homer CSE. Using the Zelen design in randomized controlled trials: debates and controversies. J Adv Nurs 2002;38:200-7.

43. Adamson J, Cockayne S, Puffer S, et al. Review of randomised trials using the post-randomised consent (Zelen's) design. Contemp Clin Trials 2006;27:305-19.

44. Eapen V, Crncec R, Walter A. Clinical outcomes of an early intervention program for preschool children with autism spectrum disorder in a community group setting. BMC Pediatr 2013;13:3.

45. Zelen M. Randomized consent designs for clinical trials: an update. Stat Med 1990;9:645-56.

46. Brown H, Prescott R. Applied mixed models in medicine. 2nd edn. Oxford: Wiley, 2006

47. Bearss $\mathrm{K}$, Johnson $\mathrm{C}$, Smith $\mathrm{T}$, et al. Effect of parent training vs parent education on behavioral problems in children with autism spectrum disorder: a randomized clinical trial. JAMA 2015;313:1524-33.

48. Pickles A, Le Couteur A, Leadbitter K, et al. Parent-mediated social communication therapy for young children with autism (PACT): long-term follow-up of a randomised controlled trial. Lancet 2016;388:2501-9.

49. Kasari C, Lawton K, Shih $\mathrm{W}$, et al. Caregiver-mediated intervention for low-resourced preschoolers with autism: an RCT. Pediatrics 2014;134:e72-9.

50. Wetherby AM, Guthrie W, Woods J, et al. Parent-implemented socia intervention for toddlers with autism: an RCT. Pediatrics 2014;134:1084-93.

51. Glasgow RE, Lichtenstein E, Marcus AC. Why don't we see more translation of health promotion research to practice? Rethinking the efficacy-to-effectiveness transition. Am J Public Health 2003;93:1261-7.

52. McConachie H, Parr JR, Glod M, et al. Systematic review of tools to measure outcomes for young children with autism spectrum disorder. Health Technol Assess 2015;19:1-506. 